\section{Response to Allen-Bridson and Pollock}

To The Editor-We appreciate the opportunity to respond to Allen-Bridson and Pollock and thank them for their thoughtful letter. We value the data provided in their letter, which helps to illustrate that the overall under-ascertainment of clinically meaningful catheter-associated urinary tract infection (CAUTI) is likely to be lower than we reported in our study, which was purposively constrained to urinary tractrelated bloodstream infection. We also recognize that surveillance and clinical definitions are used for different purposes and may not always align.

We would, however, like to raise the following salient points. First, although the Centers for Disease Control and Prevention (CDC) National Health Safety Network (NHSN) CAUTI measures are surveillance based, they are widely used for quality improvement assessment, are tied to compensation, and are often used interchangeably as clinical CAUTI events deemed "potentially preventable." Thus, it is important for the surveillance measures to be considered clinically relevant.

Second, using historical data containing CAUTIs reported under previous definitions, the CDC reported that the recent change in the CAUTI definition led to a drop in the CAUTI standardized infection ratio to 0.55 , with a corresponding reduction in number of attributable events. ${ }^{1}$ Many hospitals might mistakenly view this reduction based purely on definitional change as improvement, thereby no longer focusing on inappropriate urinary catheter use. Additionally, the capture of data regarding previously attributed bacteremic CAUTIs through reclassification as central-line bloodstream infection (CLABSIs) denotes a shift in diagnosis and not necessarily an improved diagnosis of CLABSI. The NHSN CLABSI definition is one of exclusion; thus, bacteremia with no other primary source defined by NHSN often ends up being labeled CLABSI. Attributing bacteremia due to urinary tract infection as CLABSI may pose issues because the preventive and therapeutic measures for CAUTI and CLABSI differ.

Third, we agree that with a clinician-based approach, it is difficult to objectively measure for accuracy of diagnosis. However, patients labeled by clinicians as having CAUTI that receive antimicrobials represent a population that includes symptomatic CAUTI and asymptomatic bacteriuria. These are both relevant safety issues for patients in the hospital setting: one is related to device harm and the other is associated with unnecessary antimicrobial harm.

Finally, our conclusion was to consider alternative modifications to the CAUTI surveillance definition; clinician-based diagnosis was provided as one possible example. We further advocate the use of the device utilization ratio as an objective measure that reflects all potential risks (infectious and non-infectious) associated with the urinary catheter. ${ }^{2}$

We applaud the CDC for their efforts in enhancing patient safety. Although we are not promoting clinician-based diagnosis of CAUTI as the panacea, we believe that taking it and other alternatives into consideration have a place in modifying and improving the CAUTI definition. While this process will require several iterations before deriving a surveillance definition that best represents the clinical practice of CAUTI prevention and care, it will be a worthwhile pursuit because it will provide an incredibly powerful tool that can be used to both prevent CAUTI and reduce inappropriate antimicrobial use.

\section{ACKNOWLEDGMENTS}

Financial support: This work was supported by a Veterans Affairs Clinical Sciences Research \& Development Merit Review Award (grant no. EPID-011-11S).

Potential conflicts of interest: The authors report no conflicts of interest in relation to this letter.

\section{Todd Greene, $\mathrm{PhD}, \mathrm{MPH} ;{ }^{1,2,3}$ Mohamad G. Fakih, MD, MPH; Sanjay Saint, MD, MPH ${ }^{1,2,3}$}

Affiliations: 1. Department of Internal Medicine, University of Michigan Medical School, Ann Arbor, Michigan; 2. VA/UM Patient Safety Enhancement Program, Ann Arbor, Michigan; 3. VA Ann Arbor Healthcare System, Ann Arbor, Michigan; 4. St. John Hospital and Medical Center, Detroit, Michigan.

Address correspondence to M. Todd Greene, PhD, MPH, Epidemiologist \& Research Investigator, Ann Arbor VA Health System, University of Michigan Health System Division of General Medicine, Program Manager, VA/UM Patient Safety Enhancement Program, 2800 Plymouth Rd., NCRC Building 16, Room 470C, Ann Arbor, MI 48109 (mtgreene@med.umich.edu). Infect Control Hosp Epidemiol 2016;37:1122-1122

(C) 2016 by The Society for Healthcare Epidemiology of America. All rights reserved. 0899-823X/2016/3709-0022. DOI: 10.1017/ice.2016.143

\section{REFERENCES}

1. National Health Saftety Network e-News. 2015;10(4):6. The Centers for Disease Control and Prevention website. http://www. cdc.gov/nhsn/pdfs/newsletters/nhsn-enewsletter_dec-2015_final.pdf. Published 2015. Accessed June 3, 2016.

2. Fakih MG, Gould CV, Trautner BW, et al. Beyond infection: device utilization ratio as a performance measure for urinary catheter harm. Infect Control Hosp Epidemiol 2016;37:327-333.

\section{Accounting for Competing Events in Multivariate Analyses of Hospital-Acquired Infection Risk Factors}

To the Editor-We congratulate Brown et $\mathrm{al}^{1}$ for the excellent review article about the necessary issues that need to be addressed in multivariate analyses of hospital-acquired infection (HAI) risk factors. We agree that 4 statistical issues should 
TA B LE 1. Overview of Rate and Risk Metric Models in Multivariate Analyses of Hospital-Acquired Infection (HAI) Risk Factors

\begin{tabular}{|c|c|c|}
\hline Rate Metric Measures & Rate Metric Models & Rate Metric Issues \\
\hline HAI hazard ratios & $\begin{array}{l}\text { Cox proportional hazards regression } \\
\text { model }\end{array}$ & $\begin{array}{l}\text { - Accounts for time-at-risk } \\
\text { - Allows for time-dependent exposures } \\
\text { - HAI hazard ratios cannot be interpreted as a } \\
\text { comparison of cumulative HAI risks. } \\
\text { - Incomplete; the impact of risk factors on death/ } \\
\text { discharge without HAI is missing. }\end{array}$ \\
\hline $\begin{array}{l}\text { Event-specific hazard ratios (HAI, discharge } \\
\text { and death without } \mathrm{HAI} \text { ) }\end{array}$ & $\begin{array}{l}\text { Event-specific Cox proportional } \\
\text { hazards regression models }\end{array}$ & $\begin{array}{l}\text { - There are } 3 \text { hazard ratios for each risk factor. } \\
\text { - The HAI hazard ratios are exactly the same as } \\
\text { listed above. } \\
\text { - Provides deep insights into direct and indirect effects } \\
\text { due to competing events } \\
\text { - Allows for time-dependent exposures } \\
\text { - HAI hazard ratios cannot be interpreted as a } \\
\text { comparison of cumulative HAI risks. }\end{array}$ \\
\hline
\end{tabular}

Risk Metric Measures

Overall risk odds ratio ignoring time at risk

- Risk, cumulative risk, cumulative incidence function, Subdistribution hazard ratios

\section{Risk Metric Models}

Simple logistic regression model

Fine and Gray regression model (adapted Cox proportional hazards regression model)

\section{Risk Metric Issues}

- Simplified evaluation of overall HAI risk

- Time at risk is ignored.

- Cannot include time-dependent exposures

- Subdistribution hazard is directly linked to the cumulative HAI risk.

- Subdistribution HAI hazard ratios can be interpreted as a comparison of cumulative HAI risks.

- Inclusion of time-dependent exposures is difficult.

- It is a summary measure of all competing events.

- Probability interpretation be addressed in such an analysis as follows: (1) adjust for the at-risk time, (2) avoid the time-dependent bias in case of timedependent exposures, (3) include ecological exposure measures, and (4) account for correlated outcomes.

In addition to these challenges, we would like to emphasize the need to account for competing events when evaluating the risk of HAI. ${ }^{2,3}$ When studying the time to HAI, patients are usually followed until the occurrence of HAI, discharge, or death without HAI. Due to the definition of HAI, the risk of acquiring HAI after discharge is 0 , and it is also 0 after death. Thus, it is recognized that discharge or death without HAI are competing events for HAI. ${ }^{2,3}$ In the presence of competing events, 2 different metrics should be distinguished: the (hazard) rate metric, which explores the etiology, and the risk metric, which is related to prediction (Table 1).

For instance, in a multilevel competing risk analysis, ${ }^{4}$ we showed that intensive care unit (ICU) patients who received antibiotic treatment $48 \mathrm{~h}$ before and/or after ICU admission had a lower hazard of acquiring a primary or secondary nosocomial bacteremia of any pathogen (hazard ratio [HR], 0.83 ; 95\% CI, 0.77-0.88). For the competing events, antibiotic treatment is also associated with an increased death hazard (HR, 1.08; 95\% CI, 1.04-1.13) and a reduced discharge hazard (HR, 0.70; 95\% CI, 0.69-0.71), meaning that patients with antibiotic treatment remain at risk longer. These 3 hazard ratios refer to the rate metric. However, the infection hazard ratio cannot be interpreted as a comparison of cumulative infection risk because the cumulative infection risk depends also on all competing event hazards. ${ }^{2,3,5}$ This can be seen if the antibiotic treatment is studied on a risk metric where the subdistribution hazard ratio is 1.01 (95\% CI, 0.95-1.09). In this case, a simpler risk metric analysis via logistic regression and risk odds ratios yielded similar results. ${ }^{4}$ The phenomenon can be explained as follows: Even though patients with antibiotic treatment acquire less nosocomial bacteremia per ICU day at risk (nosocomial bacteremia HR, 0.83), their extended at-risk time in the ICU (discharge HR, 0.70) results eventually in an equal cumulative infection risk. Notably, the discharge hazard is much larger than the death hazard and is therefore the main determinant for the at-risk time.

It is very likely that there is a similar phenomenon in the analysis of Brown et al. ${ }^{1}$ In their second sensitivity analysis, 
they reported an amplified effect of direct antibiotic use in terms of an odds ratio of 2.26 (95\% CI, 1.71-2.97); this analysis was based on a risk metric (see Table 1). We believe that this amplification can be explained analogously by a competing risk analysis. As in our analysis, we expect that patients with direct antibiotic use remain at risk longer in the hospital (ie, a reducing effect of antibiotic treatment on the discharge hazard occurs without HAI).

As correctly stated by Brown et al, when analyzing cohort studies with time-fixed or time-dependent exposures using the corresponding Cox proportional hazard model (approaches 1 and 2 in Brown et $\mathrm{al}^{1}$ ), patients were technically considered censored if they experienced discharge or death without infection. This analysis is valid, but we argue that it is incomplete if the impact of the exposures on discharge or death without infection is not studied. Therefore, an additional analysis regarding the competing events is necessary. This is done by performing additional Cox proportional hazard models with the same exposures but for the competing events as the outcome. Patients who acquire a HAI are then censored at the time of infection onset. ${ }^{4}$

Such competing risk analyses are not only very informative, they might also explain phenomena due to the 2 metrics. We believe that competing risk analyses are necessary since ignoring the potential effect of exposures on the competing events can easily lead to incorrect conclusions. For instance, a rate metric analysis showed no effect of burns on HAI in African children but a simple risk metric analyses showed a 3 times higher risk of HAI because children with burns remain at risk much longer in the hospital. ${ }^{3}$ The type of metric highly matters and influences the conclusion. Thus, only the use of both metrics can provide a complete picture in multivariate analyses of HAI risk factors. ${ }^{4,5}$ However, in the presence of time-dependent exposures, the rate metric approaches are very suitable, ${ }^{6}$ but risk metric approaches have still challenging limitations in their interpretation.

\section{ACKNOW LEDGMENT}

Financial support: M.W. received funding from the German Research Foundation (Deutsche Forschungsgemeinschaft) (grant no. WO 1746/1-2).

Potential conflicts of interest: The author reports no conflicts of interest relevant to this article.

\section{Martin Wolkewitz, PhD}

Affiliations: Center for Medical Biometry and Medical Informatics, Institute for Medical Biometry and Statistics, Medical Center University of Freiburg, Germany.

Address correspondence to Prof. Dr. Martin Wolkewitz, Stefan-Meier-Str. 26, 79104 Freiburg, Germany (wolke@imbi.uni-freiburg.de). Infect Control Hosp Epidemiol 2016;37:1122-1124

(C) 2016 by The Society for Healthcare Epidemiology of America. All rights reserved. 0899-823X/2016/3709-0023. DOI: 10.1017/ice.2016.162

\section{REFERENCES}

1. Brown KA, Daneman N, Stevens VW, et al. Integrating time-varying and ecological exposures into multivariate analyses of hospital-acquired infection risk factors: a review and demonstration. Infect Control Hosp Epidemiol 2016;37:411-419.

2. Wolkewitz M, Cooper BS, Bonten MJ, Barnett AG, Schumacher $\mathrm{M}$. Interpreting and comparing risks in the presence of competing events. BMJ 2014;349:g5060.

3. Schumacher M, Allignol A, Beyersmann J, Binder N, Wolkewitz M. Hospital-acquired infections-appropriate statistical treatment is urgently needed! Int J Epidemiol 2013;42:1502-1508.

4. Wolkewitz M, Cooper BS, Palomar-Martinez M, et al. Multilevel competing risk models to evaluate the risk of nosocomial infection. Crit Care 2014;18:R64.

5. Latouche A, Allignol A, Beyersmann J, Labopin M, Fine JP. A competing risks analysis should report results on all cause-specific hazards and cumulative incidence functions. J Clin Epidemiol 2013;66:648-653.

6. Wolkewitz M, Vonberg RP, Grundmann H, et al. Risk factors for the development of nosocomial pneumonia and mortality on intensive care units: application of competing risks models. Crit Care 2008;12:R44.

\section{Reply to Wolkewitz: When to Use Cumulative Risk-Based Versus Rate-Based Approaches in the Analysis of Hospital-Acquired Infection Risk Factors? That Depends on the Question}

To the Editor-We thank Dr. Wolkewitz for his thoughtful comments and clear breakdown of cumulative risk-based and rate-based measures of association in hospital acquired infection (HAI) research. We agree that a thorough understanding of the distinction between rate-based and cumulative risk-based metrics is essential for researchers performing studies of HAI risk factors.

Another way of thinking about this distinction is through the lens of the study question, which is often either etiologic or prognostic in nature. ${ }^{1}$ The objective of an etiologic research question is to assess the causal association between a risk factor and a given outcome. That is, if a given exposure were introduced experimentally, would a given patient be more or less likely to experience the outcome. ${ }^{2}$ On the other hand, prognostic research aims to predict the probability that a patient subgroup experiences an outcome on or before a given time point in a hospital stay, irrespective of whether a given risk factor caused an increased rate of disease.

In HAI research, patient subgroups with longer hospital stays may be more likely to develop an HAI during a given stay only because of the longer average duration of their stay. Whereas a risk-based approach would capture this as a difference in cumulative risk of HAI, a rate-based approach would find that the rate of HAI is no different. Epidemiologists interested in questions regarding etiology may be more likely 